\title{
EXPERIMENTAL SEARCH FOR QUANTUM GRAVITY
}

\author{
Sabine Hossenfelder* \\ NORDITA, Roslagstullsbacken 23, 10691 Stockholm, Sweden
}

\begin{abstract}
We offer a brief survey of existent and planned experimental tests for quantum gravity. First, we outline the questions we wish to address, and then introduce some of the phenomenological models that are currently used in quantum gravity, both with and without a lowered Planck scale. After that, we summarize experimental areas where these models can be tested or constrained and discuss the status of the field.

This article is partly based on the talks at the workshop on Experimental Search for Quantum Gravity, Stockholm, July 12-16 2010.
\end{abstract}

PACS: 04.60.Bc, 11.25.Wx, 11.30.Cp

Keywords: phenomenology of quantum gravity, Lorentz-invariance violation, space-time foam, emergent gravity, deformed special relativity, causal sets, string cosmology, loop quantum cosmology, cosmic strings

\section{The Quest for Quantum Gravity}

Scientific discovery is like the mapping of unknown territory. Curiosity and the good bookkeeping of scientific inquiry gradually revealed to us the ground nature put to our feet, and while some of our knowledge will need revision upon closer investigation, today we have access to highly detailed maps and guides of nature, backed up by so much evidence we are comfortable to build our lives on this territory. But our curiosity still drives us farther, again and again pushing the boundaries of the known, venturing out into the unknown.

There are two ways the expansion of scientific knowledge, the exploration of terra incognita, goes ahead. An experiment may test a previously virgin area and make an unexpected find, leaving it for the theorist to explain and make sense of. Or, a theorist may put forward a hypothesis and make a prediction, telling the experimentalist where to look next. In physics in particular, both has historically gone hand in hand, and still does. The theorist aims to make predictions for planned experiments, and the experimentalist will be interested in testing well-founded predictions offered by the theorist. Over the centuries, we developed methods and procedures that have proven useful in this process and that we rely on today, such as peer review and repeatability of experiments. Due to the well-demonstrated "unreasonable effectiveness of mathematics in the natural sciences," [1] mathematical selfconsistency of a theory is an essential ingredient assuring quality and success.

\footnotetext{
*E-mail address: hossi@nordita.org
} 
In the following, we will focus on one particular ongoing exploration at the frontiers of our knowledge: The quest for quantum gravity.

Einstein's theory of general relativity and the standard model of particle physics together provide a remarkably successful description of our observations. But general relativity has so far refused to be quantized - it still is an entirely classical theory. Using the same methods for quantization that were successful for all the other interactions in the standard model does not bring the desired result. It's not that gravity cannot be quantized - it is possible to quantize gravity by the standard procedures - but the result is non-renormalizable and can in the best case merely be understood as an effective, not as a meaningful fundamental theory.

This issue is more than a mere annoyance for the aesthetically inclined theoretical physicist. It signals a mathematical tension and a lack of understanding. In other words, it points us into the direction of unknown territory where we can expect to gain deep insight into the workings of nature if we manage to reveal its secrets. There are three main reasons why the present status requires a solutions:

1. Quantum particles can exist in superposition states. The best known example is Schrödinger's cat which is neither dead nor alive, but more realistically a photon traversing a double-slit that is neither here nor there. These animals, or particles respectively, carry energy and thus gravitate. Yet we do not know what their gravitational field is: as a classical field it does not exist in superpositions.

2. General relativity predicts the formation of singularities; instances of infinite energy density and gravitational forces. Such singularities are unphysical and signal a breakdown of the theory. In these extreme areas of spacetime, general relativity would have to be replaced with a more fundamental theory.

3. The black hole information loss problem. Using quantum field theory in a classical black hole geometry, Hawking [2] showed that black holes emit thermal radiation and thereby lose mass. If this radiation remained thermal until the black hole was entirely evaporated, then any distribution with the same initial mass that collapsed to a black hole would eventually be converted into the same thermal final state. Detailed information contained in the initial configuration would have gotten lost. Such an irreversible loss of information however is incompatible with quantum mechanics. One hopes that a proper quantization of gravity will solve this contradiction that arises by combining quantum field theory with general relativity 1 .

The two latter arguments are based on weaknesses in our current theories that could be solved by a theory of quantum gravity. It is far from clear whether a theory of quantum gravity would solve these puzzles, but it is plausible to expect that it could give us a clue how to proceed. The first mentioned problem with superposition states is a stronger argument in that its solution necessitates quantum gravity.

\footnotetext{
${ }^{1}$ This hope is not without problems either, but further discussion would lead us astray. One can also argue that the problem with singularities and that of black hole information loss are actually the same problem, see [3].
} 
The phenomenology of quantum gravity, which this article is dedicated to, is a still young research field exploring a possibility which has previously been neglected in our quest: the possibility to extract knowledge about the unknown, looked-for theory from experiment. In the following section, we will survey some of the phenomenological models used and, in section 3., the experimental possibilities to test them. In section 4. we will look at the possibility that evidence for quantum gravity may be contained in already available data.

This article is not a review. Rather, it is a introduction into the main concepts and ideas of phenomenological quantum gravity. The brief summary provided here, and the literature references, are thus necessarily incomplete and I wish to apologize in advance for every contribution to the research in this field which did not find a place here. I invite the interested reader to send me suggestions for improvement.

\section{Phenomenological Models}

In this section, we will get to know some of the currently used models for a phenomenology of quantum gravity. The first requirement for a good phenomenological model is of course that it be in agreement with already available data and that it be internally consistent. Most of the models that are presently used are quite young and unresolved issues exist. This makes research in this area lively, and full of discussion and controversy. We will however here not go into the details of these discussions, but take an agnostic point of view in presenting these models as proposals to open the area of quantum gravity for experimental test.

\subsection{The Planck Scale}

The scale at which effects of quantum gravity are expected to become relevant is the Planck scale, named after Max Planck who first introduced these units of energy, length and time in 1899 [4]. There is an easy way to estimate the Planck scale. Consider concentrating an amount of energy, $E$, in a volume of size $\Delta x^{3}$. Via Einstein's field equations we know that the curvature of spacetime, a second derivative of the metric, $g$, is related to the energy density. The typical perturbation that is then caused by the energy is

$$
\frac{\delta g}{\Delta x^{2}} \approx \frac{G E}{c^{4} \Delta x^{3}},
$$

where $G$ is Newton's constant. We now consider the energy to be localized as good as quantum mechanics possibly allows us, i.e. to its Compton wavelength $c \Delta x=\hbar / E$. Then one has

$$
\delta g \approx \frac{G E^{2}}{c^{3} \hbar}
$$

This distortion will become non-negligible when $\delta g \approx 1$, which happens at a particular energy scale, or mass respectively. In numbers, this mass scale, which corresponds to the Planck mass, and the related Compton wavelength, the Planck length, are

$$
m_{\mathrm{p}}=\sqrt{\frac{\hbar}{G c}} \approx 10^{16} \mathrm{TeV}, l_{\mathrm{p}}=\sqrt{\frac{\hbar G}{c^{3}}} \approx 10^{-20} \mathrm{fm},
$$


and the Planck time is $t_{\mathrm{p}}=l_{\mathrm{p}} / c$. In the following we will use units with $\hbar=c=1$, such that $l_{\mathrm{p}}=t_{\mathrm{p}}=1 / m_{\mathrm{p}}$. For other intuitive arguments why the Planck scale marks the regime in which effects of quantum gravity become important, see the nice article [5].

Compared to typical energy scales we are able to reach in our experiments, the Planck energy is extremely high, too high to hope to be able to test it directly. This simple fact, a consequence of the gravitational interaction being so weak compared to the other interactions, is what makes it so difficult to test quantum gravity. A collider to test quantum gravitational effects would need to have the size of our galaxy, and even a detector the size of planet Jupiter wouldn't measure as much as a single graviton in the lifetime of the universe [6]. This even lead Freeman Dyson to hypothesize that the regime in which the standard model of particle physics and general relativity lead to conflicting results is entirely undetectable [7]. However, in the following we will see that the situation is so hopeless not, for there are ways to reach the required precision that allows to test some models of quantum gravity phenomenology.

It is important to note here that our above estimate for the relevance of quantum effects relies on a crucial assumption. To obtain the Planck scale, we have extrapolated the gravitational interaction from the regime where we have access to experimental tests, at around $1 \mathrm{TeV}$, to about $10^{16} \mathrm{TeV}$. In our simple argument, this has entered through the use of Eq. (1) which is a consequence of general relativity. But that's 16 orders of magnitude in which unexpected new physics can start to play a role, thereby significantly altering the typical distortion the energy $E$ causes in the space-time geometry. If the gravitational interaction was modified at distances smaller than we have tested yet, distances that we have so far not been able to resolve with scattering experiments, then our extrapolation might fail, and the scale at which effects of quantum gravity become strong can be lower than what the above estimate suggests.

Scenarios where exactly this happens are models with additional compactified spatial dimensions. The relevant feature that these models (discussed in 2.4.9. and 2.4.10.) have in common is that at distances beyond today's experimental reach the gravitational interaction is modified through the propagation of gravitons into the higher dimensional spacetime. As a result, the Planck scale is lowered and can, depending on the parameters of the model, in the most interesting case be accessible at the Large Hadron Collider (LHC).

Scenarios with a lowered Planck scale have a distinctively different, and easier accessible, signature. We will thus in the following discuss them separately.

\subsection{What do we mean with Quantum Gravity?}

Before we continue, let us first clarify what we are interested in, in order to outline the topics to consider. It could spring up anywhere, the surprise discovery that will turn out to guide us towards a theory of quantum gravity, but for practical purposes we will have to restrict our attention to a limited focus area that is directly targeted at quantum gravitational effects.

We will thus leave out here two big area of research that could have potential relevance for quantum gravity, that of grand unification and modifications of general relativity. The scale at which grand unification is expected to occur is close by the Planck scale, and it is a justified hope that a clue to grand unification will put us on the right track to a theory also 
containing quantum gravity, commonly called "Theory of Everything." However, we will here exclude all searches for physics beyond the standard model that do not explicitly look for quantum gravitational effects. Likewise, we will exclude all general searches for deviations from general relativity (such as Lovelock or $f(R)$ gravity, Horava-gravity, bimetric models, Einstein-Aether gravity, theories of the Brans-Dicke type, etc.) - not because such deviations could not be related to quantum gravity, but because we cannot possibly cover all these topics in this limited space, and they have been covered elsewhere in great detail. A good review on tests of general relativity can be found in [8], and one on physics beyond the standard model in [9].

Another area that will not be discussed here are analogue models for quantum gravity, that are condensed matter systems which mimic features of general relativity and quantum field theory in a curved background [10], and other examples were these similarities exist, such as the recent analogy to Hawking radiation using ultra short laser pulse filaments [11]. We will also not discuss here condensed matter systems that are dual to some form of gravity via the conjectured AdS/CFT correspondence [12]. Though these studies may turn out to be useful to better understand quantum gravity, they do not actually address the Planck scale structure of the space-time that we live in.

Further, let us be clear that with quantum gravity we do not necessarily mean a quantization of gravity. If gravity is not a fundamental interaction, but merely applicable in a classical limit, then quantizing gravitational degrees of freedom may not be the right way to go. Similarly, if quantization is only an emergent feature, or one approximately valid in the limits that we have tested, then the quantization of gravity again might not lead us to the right fundamental theory. Therefore, with quantum gravity we will mean any approach that is able to resolve the apparent tension between general relativity and quantum field theories, and to address the three problems mentioned in section 1.

\subsection{What is a Phenomenological Model?}

Let us then turn towards the type of models that we will be discussing. There are two ways to venture forward into the unknown from where we presently are.

The one way is a "top-down" approach, the attempt to develop a new theory from first principles. A common problem with this approach is that not only does it typically imply also the development of entirely new mathematical techniques, the new theory moreover also must be successfully connected back to the theories and observations we already have. This connection is presently missing for all approaches towards a fundamental theory of quantum gravity. Such top-down approaches necessarily put a strong emphasis on mathematical consistency since it is, besides inspiration, the only guide at hand.

The other way is a "bottom-up" approach, starting from the theories we already have, trying to extend them with the hope to discover a path leading forward. The problem with this approach is that without any directive advice there are very many ways to go. Bottomup approaches are typically considered to merely be bridges towards a more fundamental theory. The emphasis on mathematical consistency is thus not quite as strong since one can expect these approaches to leave unanswered questions for the fundamental theory.

Taking clues from top-down approaches and using them as particular bottom-up extensions is then a promising middle-way to take that allows us to arrive at specifically targeted 
phenomenological models. In the following, we will discuss some examples of such models that one could call "top-down inspired bottom-up approaches." While not meant to be fundamental, so constructed models aim to predict a phenomenology that tests for specific properties the fundamental theory might have. These approaches are in most cases inspired by one or the other approach towards quantum gravity, but so far rigorous derivations from a candidate theory of quantum gravity are missing.

\subsection{Examples of Phenomenological Models for Quantum Gravity}

\subsubsection{Violations of Lorentz Invariance}

One of the most frequently asked questions about quantum gravity is whether the ground state of space-time obeys Lorentz-invariance or whether this symmetry is in fact weakly broken. If Lorentz-invariance was broken, observer-independence would be explicitly violated and a preferred frame would be singled out. This frame is typically thought to be related to the restframe of the Cosmic Microwave Background (CMB) - a naturally existent and experimentally confirmed preferred frame of our universe.

Lorentz-invariance violation (LIV) has attracted a significant amount of interest because it appears in many approaches to quantum gravity, though derivations are not conclusive. There exist Lorentz-violating models inspired by string theory [13], and it had also been claimed [14] that Lorentz invariance is broken in Loop Quantum Gravity (LQG) though it was later argued that the latter calculations were based on the use of unphysical assumptions about the ground state of the theory [15]. A preferred frame appears in noncommutative geometry [16], and Lorentz-invariance is also violated in many approaches in which gauge bosons are emergent [17].

Violations of Lorentz-invariance can be realized in different ways. The most relevant distinction to draw in this class of models is whether the breaking of Lorentz-invariance is present in the matter sector of the standard model, or whether it is restricted to the gravitational sector.

A breaking of Lorentz-invariance in the purely gravitational sector is expressed by coupling a tensor field, or its derivatives respectively, to geometric quantities constructed from the metric or the curvature. In the most widely used scenario, the tensor field is a unit, timelike vector field whose direction singles out the preferred frame. An example of such a theory is Einstein-Aether theory [18]. However, as mentioned previously, such modifications of general relativity will not be discussed here.

A breaking of Lorentz-invariance in the standard model sector can be characterized in an effective field theory limit by studying additional terms to the standard model Lagrangian breaking the symmetry [19]. These additional terms have pre-factors of powers of the Planck mass and a dimensionless coefficient that should, naturally, be of order one. If the factor is constrained significantly below that, it speaks against the presence of quantum gravitational effects.

The features of the resulting theory depend on exactly which terms are present and may include birefringence (the speed of photons depending on their polarization), modification of standard model cross-sections, threshold shifts of particle reactions, and modified dis- 
persion relations (possibly depending on the type of particle) which generally take the form

$$
E^{2}-p^{2}=m^{2}+f\left(E, \vec{p}, m_{\mathrm{p}}\right)
$$

Typically, $f$ is a power series in $E / m_{\mathrm{p}}$, with the prospects for experimental tests crucially depending on the presence or absence of the first order term.

The phenomenology of LIV models is vast, which has certainly added to their popularity. It should also be noted that for local, unitary, and Lorentz-invariant quantum field theories, CPT symmetry follows [21]. Testing CPT-symmetry thus tests for Lorentz-invariance - provided the theory is local and has a unitary evolution.

A well known problem with LIV models is that the new (irrelevant) higher order terms of mass dimension 5 and higher induce by radiative corrections terms to the Lagrangian of dimension 4 or less which are ruled out already if the higher order terms had coefficients naturally expected from quantum gravity. For these models to be consistent and not in conflict with experiment already, one thus has to either believe in fine-tuning, or some mechanism that protects the symmetry of the lower dimensional operators.

We will in the following not attempt to summarize all experimental tests that have been made on LIV, but only the most stringent ones to date on higher order terms. For a more comprehensive review, the interested reader is referred to [22].

\subsubsection{Deformations of Special Relativity}

Instead of breaking Lorentz-invariance, it has also been considered the possibility that special relativity may be modified in the high energy regime without singling out a preferred frame. This idea has become known as "Deformed Special Relativity" (DSR) [23]. In these models, the modified Lorentz-transformations in momentum space have two invariants: A constant, $c$, with dimension speed and the Planck mass, $m_{\mathrm{p}}$.

DSR has been motivated by LQG, though no rigorous derivation exists to date. There are however non-rigorous arguments that DSR may emerge from a semiclassical limit of quantum gravity theories in the form of an effective field theory with an energy dependent metric [24], or that DSR (in form of a $\kappa$-Poincaré algebra) may result from a version of path integral quantization [25]. In addition it has been shown that in $2+1$ dimensional gravity coupled to matter, the gravitational degrees of freedom can be integrated out, leaving an effective field theory for the matter which is a quantum field theory on $\kappa$-Minkowski spacetime, realizing a particular version of DSR [26]. Recently, it has also been suggested that DSR could arise via Loop Quantum Cosmology (see section 2.4.7.) [27].

This class of models has some problems that are so far unresolved, most notably the correct description of multi-particle states and the formulation in position space. It has also been argued [28] that DSR generically causes non-local effects that are in conflict with the standard model. This issue is still under debate [29].

Consequences of a DSR-modification of special relativity are that, in most but not all models, the dispersion relation is modified and the speed of light becomes energydependent. The constant $c$ that is an invariant of the transformation is then the speed of light in the low-energy limit 2 . These features are shared with LIV models, but since it has

\footnotetext{
${ }^{2}$ It can also be argued that, since the Planck mass as a coupling constant should actually be energy dependent
} 
been argued that DSR may not be expressible in form of an effective field theory and to date no (agreed upon) Lagrangian formulation for the interaction picture exists, most of the constraints on LIV do not apply for DSR. It had originally been claimed that DSR leads to a shift of thresholds of particle reactions [31], but this claim has later been revised [32].

\subsubsection{Causal Sets}

The causal sets approach [33] considers as fundamental the causal structure of spacetime, as realized by a partially ordered, locally finite set of points. This set, represented by a discrete sprinkling of points, replaces the smooth background manifold of general relativity. The "Hauptvermutung" (main conjecture) of the causal sets approach is that a causal set uniquely determines the macroscopic (coarse-grained) space-time manifold. In full generality, this conjecture is so far unproven, though it has been proven in a limiting case [34].

Intriguingly, the causal sets approach to a discrete space-time can preserve Lorentzinvariance. This can be achieved by using not a regular but a random sprinkling of points. It has been shown in [35], that a Poisson process fulfills the desired property.

This discretization of the background manifold leads to a Lorentz-invariant diffusion of energy-momentum; its most general form contains two phenomenological parameters that can be bounded by the CMB spectrum [36].

\subsubsection{Minimal Length and Generalized Uncertainty}

The idea that the Planck length might play the role of a fundamentally minimal length in nature goes back to Heisenberg in 1938 [37]. And indeed, there are many indications that this is the case (for reviews see [38]). LQG has a minimal unit of area [40], in string theory it's string excitations that prevent an arbitrarily good resolution of space-time structures [39], and non-commutative geometries [16] have a minimal length scale built in already which results in a finite localization of wave-packets [41]. There are furthermore several thought experiments for probing smallest distances with test particles that lead one to conclude the non-negligible perturbations of the background geometry close by the Planck scale result in the Planck scale setting a limit to how well we can test structures [42, 30]. This is not so surprising because one would expect the Planck length to play the role of a regulator in the ultraviolet.

One can then build a model that incorporates the notion of a minimal length into quantum mechanics and quantum field theory [43]. Features of these models are a generalized uncertainty principle that prevents an arbitrarily good localization in position space, a modified dispersion relation, and a modified measure in momentum space which can be understood as a non-trivial geometry of momentum space. Such models may or may not have an energy-dependent speed of light [46]. They either break Lorentz-invariance explicitly or exhibit features akin the previously discussed DSR, albeit with a possibly different interpretation [47]. The models that break Lorentz-invariance or represent a version of DSR can be tested via these properties. Those that don't typically lead to predictions, such as modifications of quantum mechanics and cross-sections, that are far outside possible experimental

too, the second invariant constant of the transformations is similarly the Planck mass in the low-energy limit [30]. 
tests (unless the Planck scale is lowered in which case they become observable in the same regime as other effects of models with a lowered Planck scale [44]). The most reasonable place to expect an observable phenomenology is from the high temperature phase in the early universe. Thus, the thermodynamical properties of these models have recently been a subject of increased attention [45], though there are no predictions yet.

It is important to note that one does not need to have a discrete spacetime to have a fundamentally finite resolution of that spacetime and thus, by employing these models, one does not subscribe to a particular space-time picture.

\subsubsection{Space-time foam and granularity}

A central mystery of quantum gravity is the structure of space-time on Planckian scales. While the details might not be known, the expectation that it is subject to quantum fluctuations that on shortest distances significantly distort the background which on long distances seems so smooth, has become known as "space-time foam." In such models, the background itself is as usual described by a metric. There are then different ways to model such fluctuations of the background, depending on the type of deviations from flat space that one considers.

One consequence of such models is that the departures of the background geometry from flat space on short distances can cause quantum mechanically pure states to evolve into mixed states [48]. This quantum gravitationally caused decoherence, effectively incorporated in a non-unitary quantum mechanical time-evolution leads to violations of CPT invariance. Another feature are fluctuations of light-cones, leading to a stochastic rather than a systematic departure from a constant speed of light.

An entirely different approach to think about space-time on Planckian scales is that of a granular structure [49] which respects Lorentz-invariance. In this model, the effect of the space-time structure is argued to appear in a non-trivial coupling between the Weyl-tensor and the standard model sector.

\subsubsection{Geometrogenesis}

If space-time is fundamentally discrete and described by a network, we find this network today in a very orderly and highly special state that is well approximated by a smooth manifold. But one would expect the orderliness of this state to depend on the temperature, with the network being in a completely different state at high temperatures in the early universe, and a manifold-like structure only emerging in a phase transition.

This idea of geometry emerging from a non-geometrical phase has been dubbed "geometrogenesis" [50], and it has been studied in a model of Quantum Graphity [51]. In this model, one assigns a Hamiltonian to the network which contains information about the node- and link-structure, and tries to find the ground state and the temperature dependence of the network's regularities. This model is still work in progress, but one can hope that in the soon future it will lead to distinct predictions in the areas of cosmology and astrophysics.

It has been shown in [52] that a phase transition from a holographic pre-geometric phase at high temperatures is, under quite general conditions, compatible with present day observation. 


\subsubsection{Loop Quantum Cosmology}

Loop Quantum Cosmology (LQC) [53] incorporates implications for the quantum geometry derived from LQG in cosmological settings such that, for sufficiently general parameterizations of the expected effects, the freedom for instance in quantization choices is restricted. LQC is basically a symmetry-reduced version of LQG and in this way provides a way to test the consistency of the full theory. Currently, cosmological applications are at a conceptual level, such as suggestions to resolve the singularity problem, but derivations are moving closer to making contact with potential observations.

In the context of the singularity problem, several mechanisms have been suggested, all related to changes in the quantum space-time geometry (rather than modifications of matter terms that might violate energy conditions). In isotropic LQC the analog of the WheelerDeWitt equation is a difference equation, which allows one to evolve the wave-function through geometrical configurations that correspond to the classical singularity [54]. Several models (especially those whose matter energy is dominated by the kinetic term of a scalar field) exhibit bouncing solutions for their wave-functions [55]. Once inhomogeneities can be evolved consistently through the bounce of a universe model, one may expect interesting phenomenological implications. However, at present the required perturbation theory is not sufficiently developed to consider inhomogeneities in regimes of strong quantum corrections that are required to trigger a bounce.

For phenomenology, linear perturbations around spatially flat Friedmann-RobertsonWalker models have been considered for small quantum corrections, and cosmological perturbation equations are now available without having to use gauge-fixing of space-time diffeomorphisms [56], which allows one to draw conclusions about the structure of spacetime underlying LQG. Interestingly, it turns out that the quantum corrections cannot merely come from higher-curvature terms of an effective action, because not just the dynamics but also the underlying gauge algebra changes. This deformed gauge algebra can be interpreted as a realization of DSR [27]. The impossibility to express the corrections as highercurvature terms means that quantum corrections can show signatures characteristic of the quantum-geometry corrections of LQC. Phenomenological implications are presently being explored mainly for tensor modes [57] but also the more complicated scalar modes are getting under better control.

\subsubsection{String Cosmology}

String cosmology is a class of models for the early universe that incorporate key principles of superstring theory, such as dilatonic (and axionic) forces, duality symmetries, winding modes, limiting sizes and curvatures, and higher-dimensional interactions among elementary extended objects. The hope is to clarify or resolve some big problems of standard and inflationary cosmology such as the space-time singularity, the physics of the trans-Planckian regime, or the initial conditions for inflation.

There are at present four different, but related, string cosmology scenarios [58]: the prebig-bang scenario [59] (for a review see [60]), the string gas/brane gas scenario [61] (for a review see [62]), the ekpyrotic/cyclic scenario [63], and the brane-antibrane inflationary scenario [64].

In the first three cases the initial singularity is removed, and the standard cosmological 
phase is preceded in time by a new epoch dominated by string effects. The standard phase is complemented by an inflationary but low-energy phase, infinitely extended in time, and asymptotically evolving from the string perturbative vacuum. In the string gas scenario, the standard cosmological phase emerges from an initial, higher-dimensional and topologically non-trivial regime in which winding modes and momentum modes of string and branes are in dynamical equilibrium, thus preventing the cosmological expansion. A nice feature of string gas cosmology is that the gas of winding modes stabilizes the size [65] and shape [66] of the extra spatial dimensions. In the ekpyrotic scenario, an extra spatial dimension is initially shrinking, and the beginning of the standard cosmological phase is triggered by the collision and the bounce of the two domain walls representing the space-time boundaries. The absence of a singularity in these three scenarios stands in contrast to the scenario of brane-antibrane inflation, where the big bang singularity remains, and where inflation has the conventional dynamics as in standard slow-roll models.

These four scenarios do not necessarily exclude each other. For instance, the initial epoch typical of pre-big-bang models leads the background cosmological fields towards a regime of strong coupling, and could be responsible for the process of brane/antibrane production, thus setting the initial conditions for the phase of brane-antibrane inflation, or for the ekpyrotic bounce, or for the epoch of brane gas equilibrium.

For what the phenomenology is concerned, the scenario of brane-antibrane inflation leads to basically the same predictions as in conventional models of slow-roll inflation. String gas cosmology provides an alternative to cosmological inflation for explaining the origin of structure in the universe. Here, thermal fluctuations of strings in the Hagedorn phase lead to curvature perturbations which have a scale-invariant spectrum like that predicted in inflationary cosmology and the amplitude of the curvature fluctuations at late times is in good agreement with the observed value. These scenarios have further implications for cosmology that will be discussed in section 3.3 .

\subsubsection{Lowered Planck scale: The Arkani-Hamed-Dimopoulos-Dvali Model}

In the Arkani-Hamed-Dimopoulous-Dvali (ADD) model [67] (for a review, see [68]), our usual $3+1$ dimensional space-time has $d$ additional space-like dimensions, each of which is compactified on a radius of dimension $R$. All particles that carry standard model charges live on a 3-dimensional submanifold (the standard model brane) in that higher dimensional space, whereas gravitons are allowed to propagate into all dimensions (the bulk) 3 . This setting is motivated by string theory, in which gravitons are described by closed strings that are free to propagate, whereas particles with standard model charges are described by closed strings with ends attached to the brane.

This model explains in geometrical terms why gravity is so much weaker than the other interactions, a puzzle known as the "hierarchy problem." Because it is able to propagate into more dimensions, gravity dilutes much faster on short distances $(\ll R)$ than as if it was bound to the brane, and on long distances $(\gg R$ ), where we recover to good accuracy $3+1$ dimensions, gravity thus appears weakened relative to the other interaction. The relation between the fundamental, higher dimensional Planck scale, $M_{\mathrm{f}}$, and the Planck scale we

\footnotetext{
${ }^{3}$ A right-handed neutrino, should it exist, would also not be bound to the brane.
} 
have measured at long distances is given by the volume of the extra dimensions

$$
m_{\mathrm{p}}^{2}=R^{d} M_{\mathrm{f}}^{d+2} .
$$

If one inserts some numbers, assuming the fundamental scale $M_{\mathrm{f}}$ is around a $\mathrm{TeV}$, one finds that for $d>2$ the radius of the extra dimensions is far below the distances for which we have direct tests of the gravitational potential, with the case $d=2$ meanwhile being tightly constrained by precision measurements [69] (and the case $d=1$ is ruled out - the extra dimension would need to have the size of the solar system). Note however that the ADD-scenario does not actually solve the hierarchy problem, but merely reformulates it, since the inverse of the radius $R$ has to be many orders of magnitude smaller than $M_{\mathrm{f}}$, which introduces a new unexplained hierarchy.

The most important consequences of this scenario are that on short distances, gravity is stronger than without extra dimensions, and that gravitons can have a non-vanishing momentum-component into the direction of the extra dimensions. The graviton's momentum is then geometrically quantized in multiples of the inverse radius. On the brane, these graviton excitations - also referred to as "the Kaluza-Klein-tower" - appear like massive gravitons.

The ADD model has attracted a lot of attention during the last decade and exists in many variants with different radii of extra dimensions, compactifications on different topologies, or taking into account a finite width of the brane (which itself could have an internal structure), brane recoil, and many other details that we will not go into here.

\subsubsection{Lowered Planck scale: The Randall-Sundrum Model}

The Randall-Sundrum (RS)-model [70] (for a review, see [71]) equips space-time with one additional space-like dimension and, as in the ADD-model, gravitons are allowed to propagate into the full space-time, while particles with standard model charges are bound to a $3+1$ dimensional submanifold. In contrast to the ADD-model however, the geometry of the extra dimension in the RS-model is not flat but curved with an exponential function of the coordinate of the extra-dimension, the so-called "warp-factor." In the bulk, one has a negative cosmological constant, and the space-time is thus a slice of Anti de-Sitter (AdS) space with the standard-model-brane, also called the TeV-brane, on one end, and another brane called the Planck-brane on the other end. In one variant of the model (known as RS2), this slice is infinitely extended, but we will here focus on the case (RS1) where it is of a finite size $\pi R$.

The relation between the fundamental scale, $M_{\mathrm{f}}$, and the usual Planck scale is in the RS-model given by

$$
m_{\mathrm{p}}^{2}=\frac{M_{\mathrm{f}}^{3}}{k}\left(1-e^{-2 \pi k R}\right)
$$

where $k$ is the curvature radius of the AdS slice. To examine the strength of gravity on the TeV-brane, one considers the standard model Lagrangian on that brane. One finds that one can absorb all factors stemming from the non-trivial metric in field redefinitions and thus reproduce the usual Lagrangian, except that the vacuum expectation value of the Higgs is exponentially suppressed by a factor $\exp (-\pi k R)$, thus explaining the smallness of particle 
masses relative to the Planck mass. To obtain the observed value, one then has to choose the product $R k$ accordingly. By merit of the exponential function, it is here not necessary to introduce another hierarchy to achieve a factor of many orders of magnitude - it is sufficient if $k R$ is of order ten.

The wave-equations for the gravitons in the background geometry of the RS-model are Bessel equations. Their solutions lead to a discrete, though not periodic, mass spectrum for graviton excitations with a spacing of the order $k \exp (-\pi k R)$.

As the ADD-model, the RS-model meanwhile exists in many variants - different field localizations, thick branes, additional dimensions - that we will not discuss here.

\subsubsection{Other}

An interesting proposal that should be mentioned here whose origin dates back more than 30 years [72] is that of cosmic strings (for a review see [73]). Cosmic strings are $1+1$ dimensional defects formed in the early universe that, under appropriate conditions, can grow during the evolution of the universe. Cosmic strings are characterized by their energy density. Today, they would form a network of (infinitely) long strings and closed loops.

Originally considered to be caused by the breaking of axial symmetries in quantum field theories, cosmic strings have recently attained renewed interest as they could appear also as relics of fundamental strings [74]. While cosmic strings of either type would be an important discovery allowing us a glimpse into the early universe, for the sake of quantum gravity phenomenology the question is whether it would possible to distinguish fundamental strings from the quantum field theory strings.

\section{Experimental Search and Predictions}

In this section, we will summarize the most important experimental tests of the previously introduced models. Neutrino-physics will be discussed in a separate section 3.4. rather than be broken down by the various sources of neutrinos.

\subsection{Collider Searches}

The most powerful particle colliders in operation today are the Tevatron at Fermilab, and the LHC at CERN. These experiments are allowing us to peer into the structure of matter on distance scales down to $\approx 10^{-18} \mathrm{~m}$. If the Planck scale is lowered, in either the ADD- or the RS-scenario, it might become accessible in collider experiments. To solve the Hierarchy problem, one expects the "true" Planck scale to be not too much above the electroweak scale $(\approx 250 \mathrm{GeV})$, thus hopes are that the Tevatron has established lower bounds, and LHC may be able to catch a glimpse of quantum gravity.

The relevant signatures in this case are the production of gravitons, modifications of standard model cross-sections by virtual graviton exchange, and the production of black holes. The production of black holes in particular would be a spectacular observation, giving us an experimental handle on the black hole information loss problem. Present Tevatron data allows to put constraints on the parameters of both the RS- and the ADD-model, in 
particular from precision electroweak observables. For the LHC, numerous detailed predictions have been made, including many subclasses of models and specific scenarios that are presently being tested. An up-to-date summary of the best current constraints on the model parameters in both the RS and the ADD-model, including the relevant references, can be found in the Particle Data Book [75]. As one can expect, the constraints on the new fundamental scale are of the order TeV. In the ADD-scenario the exact constraints are usually dependent on the number of extra-dimensions.

\subsection{Astrophysics}

Astrophysical processes can occur at higher energies than we are presently able to achieve in particle colliders, but they happen in a less controlled experimental environment which implies additional uncertainties. For this reason, astrophysical and collider constraints often complement each other.

Next to the previously discussed constraints from collider physics, the ADD-model is also tightly bounded by astrophysical data. The emission of massive gravitons can lead to an additional cooling of supernovae [76], radiative decays of these gravitons can give rise to a diffuse cosmic gamma-ray background [77], and gravitationally trapped massive gravitons can result in a re-heating of supernovae remnants and neutron-stars [78]. These effects lead to bounds on the model's parameters that are also summarized in the Particle Data Book [75] and, for less than 4 extra dimensions, push the new fundamental scale above the range testable at the LHC. There are moreover constraints from ultra high energetic cosmic rays (UHECR), which are similar to the ones from collider physics - though the energy is somewhat higher still, additional theoretical and experimental uncertainties come in to play. With this, we will leave now the scenarios with a lowered Planck scale.

The tightest constraints on Lorentz-invariance violating higher order operators come today from astrophysics. By analyzing the electromagnetic radiation from the Crab Nebula, the relevant parameters for order 5 terms in an extension of quantum electrodynamics [79] have been constrained to $\xi<\mathcal{O}\left(10^{-7}\right)$ and $\left|\eta_{ \pm}\right|<\mathcal{O}\left(10^{-5}\right)$ [80]. We remind the reader that the natural value one would expect from quantum gravitational effects is of order one.

One of the predictions of DSR that is presently tested is that of an energy-dependence in the arrival time of highly energetic photons from distant $\gamma$-ray-bursts (GRB) [81]. Such an effect can also arise from LIV but, as previously mentioned, LIV models are tightly constrained already, whereas DSR evades these constraints and thus a parameter $\alpha$ of order one for first order deviations in the dispersion relation is still under consideration. The timedelay $\Delta T$ one expects between two photons with an energy difference $\Delta E$ from Eq.(4) for such a first-order deviation is

$$
\Delta T=\alpha \frac{\Delta E}{m_{\mathrm{p}}} L,
$$

where $L$ is the distance the photons have travelled. If one inserts some numbers, one finds that the delay can be of the order seconds for a distance of some $\mathrm{Gpc}$ and some $\mathrm{GeV}$ of energy, if $\alpha$ is of order one. The best constraint to date comes from the GRB090510, which puts the limit at $\alpha<1.2$ for the case in which higher energetic photons are slowed down [82]. It is significantly harder to put bounds on a scenario in which the effect is stochastic, and better statistic is needed for that. 
It has also been suggested that a modified dispersion relation as it appears in LIV and DSR may be tested with weak gravitational lensing [81, 83], but the effect is out of presently possible precision.

Cosmic strings, should they exist, would cause particular gravitational lensing images that however would not allow us to distinguish whether these strings are of fundamental origin. More interesting for our purposes is thus that cosmic strings would generically create cusps and be sources of gravitational radiation. This could be detected in the soon future using experiments like LIGO or LISA. In the case of the fundamental cosmic super-strings that are interesting for quantum gravity phenomenology, the presence of extra dimensions enhances the number density of loops in the strings' network [84]. It has however been pointed out recently that taking into account the full dynamics of the string in presence of extra dimensions larger than the width of the string also has the effect of lowering the probability of cusp formation and of dampening the gravitational wave emission, thus making the detection more difficult [85].

Light-cone fluctuations that appear in models of space-time fuzziness can lead to spectral line broadening and angular blurring of distant sources [86]. This effect too is unfortunately not detectable with presently possible precision.

\subsection{Cosmology}

The best place to look for quantum gravitational effects is in regions of strong curvature, i.e. towards the center of black holes or the early universe. Since the black hole interior is hidden from observation by the horizon, this makes the early universe the prime candidate.

Our currently most remarkable insights about this era stem from the CMB whose temperature anisotropies carry an imprint of the background perturbations back then. The CMB radiation is polarized, with the polarization pattern commonly decomposed into $E$-modes (curl-free) and $B$-modes (divergence-free), in an analogy to electrodynamics. The detection of $B$-modes provides a signature of primordial gravitational waves (tensor modes) and via those contains information about the dynamics of the background. The precision of $B$-mode measurements is expected to significantly increase in the soon future through experiments like QUaD, BICEP, ABS, and Quijote. For the following, it is useful to know that in the standard (slow-roll, single field) inflationary scenario the quantum fluctuations

of the metric tensor have a flat or almost flat spectrum, and the CMB-fluctuations are to good precision Gaussian.

In the pre-big-bang scenario of string cosmology, the quantum fluctuations of the metric tensor tend to be amplified with a spectrum which is rapidly growing in frequency. This has two important physical consequences. First, the rapidly growing spectrum of the tensor metric perturbations leads to a relic background of pre-big-bang gravitons which peaks at high frequency [87]. This signature should be easily accessible to planned experiments for Earth-based detectors (e.g. LIGO, VIRGO) and gravitational antennas operating in space (e.g. LISA, BBO, DECIGO). At the low frequency scales relevant to the observed CMBanisotropy, the spectrum is in contrast strongly suppressed and a possible contribution to the CMB- polarization should be completely negligible [88]. Second, the non-standard production of CMB-anisotropies in the pre-big-bang scenario induce a small non-Gaussianity in the CMB-spectrum, which may be detectable in the soon future: with the WMAP 8-year 
mission results one expects a $20 \%$ improvement on the bounds on non-Gaussianity, while ESA's Planck satellite can yield a factor of about 4.

In the ekpyrotic string cosmology scenario the relic background of primordial gravitons also has a growing spectrum, but is expected to be quite negligible today both in the high-frequency and low-frequency range [89]. In string gas cosmology, the spectrum of gravitational waves is predicted to be mostly scale-invariant but also with a slight blue tilt [90]. The scenario of brane-antibrane inflation finally basically leads to the same predictions as in conventional models of slow-roll inflation.

In LQC, scenarios without a bounce produce a tensor mode spectrum quite similar to that in the ekpyrotic string cosmology scenario [57]. In case of a bounce, the power of tensor modes has also been found to be suppressed at small frequencies relative to the standard scenario, but with a characteristic bump at an intermediate scale and approaching the standard result at high frequencies [91]. The model parameters of this prediction (the mass of the inflaton field and the position of the peak in the spectrum) were recently constrained with presently available data on the CMB $B$-modes from QuAD and BICEP in [92]. One can expect these constraints to tighten significantly with better data.

Cosmic strings are yet another mechanism to produce non-Gaussianities [93], and they also affect the spectrum of CMB $B$-modes [92], both of which yields constraints on their abundance.

The Lorentz-invariant diffusion that appears in a model inspired by the causal sets approach also affects the polarization of CMB photons [94]. Presently available data was found to be consistent with no effect, though with a slight bias for a small effect.

\subsection{Neutrino Physics}

Neutrinos are interesting for the purposes of testing feeble quantum gravitational effects for two reasons. First, their very small masses and second, their weak interaction which enables them even at high energies to travel long, possibly cosmological, distances almost undisturbed. Neutrino experiments fall into different categories, depending on the source of neutrinos: Earth based (reactor, collider, and other man-made neutrino sources), solar neutrinos, atmospheric neutrinos, and cosmogenic neutrinos. From those, the latter reach the highest energies and longest travel times, exceeding $10^{20} \mathrm{eV}$ and some Gpc distance. On the other hand, their flux is small at high energies, such that collecting useful statistics is difficult.

In [95], it has been suggested to use cosmogenic neutrinos to tighten bounds on LIV with upcoming experiments. In [96] it has been pointed out that cosmogenic neutrinos could be used to test modified dispersion relations to high precision if a baseline of $L \sim \pi m_{\mathrm{p}}^{4} / E^{5}$ could be reached, where the flux-ratio of different neutrino species would become sensitive to the distance. And though the energies and distances needed are so far not achievable, technological progress in that area is rapid. Cosmogenic neutrinos might at some point also be able to constrain scenarios with a gravitationally induced collapse of the wave-function [97].

In some models, CPT-violation can be a cause of neutrino-oscillation (rather than a mass-difference) [98], though the simplest models are already ruled out by data [99]. Such scenarios have the additional virtue of possibly accommodating the LSND and MiniBoone 
data [100]. Their challenge is typically to be able to fit all the present data without introducing too many parameters.

It has further been proposed to combine neutrino measurements with the detection of photons from $\gamma$-ray bursts to better constrain modified dispersion relations in DSR and LIV [101].

\subsection{Other}

Among the effects that do not fall into any of the above categories counts for example a particular sort of holographic noise induced by space-time fluctuations that may be detectable in presently operating gravitational wave interferometers [102]. The model of space-time granularity (2.4.5.) is testable in an Eötvös-like experiment [103] and might soon be subject to constraints. Finally, it should be mentioned the neutral Kaon doublet whose oscillations allow for tests of LIV and CPT-violation induced by decoherence. The precision of these tests for decoherence is presently at the level where one expects effects of quantum gravity to become important if the relevant parameters have natural values of order one [104].

\section{Postdictions}

In the previous section, we have summarized so far unexplored areas in which we might be able to find signal of quantum gravity, at higher energies, better precision, larger distances or combinations thereof. But there is also the possibility that we have already seen signatures of quantum gravity, yet not recognized them for what they are for we have today experimental data which is insufficiently understood, and thus points at shortcomings in our current theories.

The most obvious shortcoming in our ability to explain available data we are facing in cosmology. An increasing amount of cosmological and astrophysical observations with ever better precision show that $96 \%$ of the universe's content has peculiar properties, different to those of matter we are made of. Approximately 74\% is "dark energy" and $22 \%$ is "dark matter" [105]. Dark energy obeys an unusual equation of state that has the consequence of the universe's expansion accelerating. The equation of state of dark matter is that of our usual matter but, as dark energy, it is non-luminous and thus, so far, evading direct detection. While we can describe the behavior of dark energy and dark matter on a macroscopic level for the purposes of cosmology and astrophysics, we do not presently know their microscopic origin. Proposals for both are abundant.

Other unexplained data is rather banally the masses of elementary particles. Why they have the values we observe nobody knows. Likewise nobody knows why the particles classify in three generations, or why we live in $3+1$ dimensions.

It might be that some of these puzzles find their answer in quantum gravity. Just to mention a few ideas in this area: The cosmological constant for example might be a result of a specific sort of defects in a discrete space-time slicing, which are non-local links connecting points that have a macroscopic distance in the background manifold [106]. In [107] it was put forward a proposal after which neutrino-masses and the cosmological constant are emergent, with additional predictions of Lorentz- and CPT-violating neutrino oscillations. The cosmological constant has also a natural place in the causal sets approach [108]. It has 
further been suggested that a string theory inspired model might be an alternative to dark matter when it comes to galaxy rotation curves [109], and string gas cosmology provides a mechanism for explaining why only three of the nine spatial dimensions which string theory contains can become macroscopic [110].

We will certainly learn more about the viability of these proposals with running or upcoming direct searches for dark matter and more precise tests for the equation of state of dark energy, for example from high-redshift supernovae.

\section{Summary}

The phenomenology of quantum gravity is a young and lively research field that brings together theoretical and experimental physicists from many areas. As such, it faces particular challenges. One is that the predictions of different models may be very similar. To address this issue, the various predictions have to be contrasting directly, possibly combining several experimental areas. This has been done very well with the LHC predictions from extra dimensional models, but similar efforts are presently lacking for example when it comes to different scenarios for the early universe. The other challenge is in the collaboration and communication between theory and experiment, an exchange that becomes ever more important the more involved data analysis becomes.

To date we have no experimental signature for quantum gravitational effects. But, as we have seen in the previous sections, creativity, persistence and technological improvements have brought us closer to this goal. The quest for quantum gravity may proceed slowly and sometimes be frustrating, but the reward will be nothing less than a revolution of our understanding of space and time.

\section{Acknowledgements}

I thank Ed Copeland, Ruth Gregory and Stefan Scherer for clarifying discussions, and I am especially grateful to Robert Brandenberger, Martin Bojowald, Maurizio Gasperini and Lee Smolin for their helpful contributions to the work on this manuscript. I further thank all participants of the workshop on Experimental Search for Quantum Gravity that took place at NORDITA, Stockholm, July 12-16, 2010. The interested reader can find recordings of the talks at www. nordita.org/esqg 2010.

\section{References}

[1] E. Wigner, Comm. Pure Appl. Math. 13 (1): 114 (1960).

[2] S. W. Hawking, Commun. Math. Phys. 43, 199 (1975) [Erratum-ibid. 46, 206 (1976)]; Phys. Rev. D 14, 2460 (1976).

[3] S. Hossenfelder and L. Smolin, Phys. Rev. D 81, 064009 (2010) [arXiv:0901.3156 [gr-qc]].

[4] M. Planck, Sitzungsberichte der Königlich Preußischen Akademie der Wisseschaften zu Berlin 1899 - Erster Halbband (Berlin: Verl. d. Kgl. Akad. d. Wiss. 1899).

[5] R. J. Adler, arXiv:1001.1205 [gr-qc]. 
[6] T. Rothman and S. Boughn, Found. Phys. 36, 1801 (2006) [arXiv:gr-qc/0601043].

[7] F. Dyson, in “The Scientist as Rebel,” New York Review Books 2006.

[8] C. M. Will, Living Rev. Rel. 9, 3 (2005) [arXiv:gr-qc/0510072].

[9] B. C. Allanach et al., arXiv:hep-ph/0602198.

[10] C. Barcelo, S. Liberati and M. Visser, Living Rev. Rel. 8, 12 (2005) [arXiv:gr-qc/0505065];

R. Schutzhold, Class. Quant. Grav. 25, 114011 (2008) [arXiv:1004.2586 [gr-qc]].

[11] F. Belgiorno et al., arXiv:1009.4634 [gr-qc].

[12] H. Nastase, arXiv:0712.0689 [hep-th]; S. Sachdev, arXiv:1002.2947 [hep-th].

[13] V. A. Kostelecky and S. Samuel, Phys. Rev. D 39, 683 (1989); J. R. Ellis, N. E. Mavromatos and D. V. Nanopoulos, arXiv:gr-qc/9909085, N. E. Mavromatos, PoS QG-PH, 027 (2007) [arXiv:0708.2250 [hep-th]]; C. P. Burgess, J. M. Cline, E. Filotas, J. Matias and G. D. Moore, JHEP 0203, 043 (2002) [arXiv:hep-ph/0201082].

[14] R. Gambini and J. Pullin, Phys. Rev. D 59, 124021 (1999) [arXiv:gr-qc/9809038].

[15] L. Smolin, private communication.

[16] M. R. Douglas and N. A. Nekrasov, Rev. Mod. Phys. 73, 977 (2001) [arXiv:hep-th/0106048].

[17] J. Bjorken, arXiv:hep-th/0111196, P. Kraus and E. T. Tomboulis, Phys. Rev. D 66, 045015 (2002) [arXiv:hep-th/0203221]; A. Jenkins, Phys. Rev. D 69, 105007 (2004) [arXiv:hep-th/0311127]; J. L. Chkareuli, C. D. Froggatt, R. N. Mohapatra and H. B. Nielsen, arXiv:hep-th/0412225

[18] T. Jacobson and D. Mattingly, Phys. Rev. D 64, 024028 (2001) [arXiv:gr-qc/0007031]; T. Jacobson, PoS QG-PH, 020 (2007) [arXiv:0801.1547 [gr-qc]].

[19] D. Colladay and V. A. Kostelecky, Phys. Rev. D 55, 6760 (1997) arXiv:hep-ph/9703464];

[20] D. Colladay and V. A. Kostelecky, Phys. Rev. D 58, 116002 (1998) [arXiv:hep-ph/9809521].

[21] G. Luders, Annals Phys. 2, 1 (1957) [Annals Phys. 281, 1004 (2000)].

[22] D. Mattingly, Living Rev. Rel. 8, 5 (2005) [arXiv:gr-qc/0502097].

[23] G. Amelino-Camelia, Phys. Lett. B 510, 255 (2001) [arXiv:hep-th/0012238]; J. KowalskiGlikman, Phys. Lett. A 286, 391 (2001) [arXiv:hep-th/0102098]; G. Amelino-Camelia, Nature 418, 34 (2002) [arXiv:gr-qc/0207049]; J. Magueijo and L. Smolin, Phys. Rev. D 67, 044017 (2003) [arXiv:gr-qc/0207085].

[24] G. Amelino-Camelia, L. Smolin and A. Starodubtsev, Class. Quant. Grav. 21, 3095 (2004) [arXiv:hep-th/0306134]; L. Smolin, arXiv:0808.3765 [hep-th].

[25] J. Kowalski-Glikman and A. Starodubtsev, Phys. Rev. D 78, 084039 (2008) arXiv:0808.2613 [gr-qc]].

[26] L. Freidel, J. Kowalski-Glikman and L. Smolin, Phys. Rev. D 69, 044001 (2004) [arXiv:hep-th/0307085.

[27] M. Bojowald, "LQGD(S)R: On the low-energy implications of loop quantum gravity", talk delivered at the workshop on Experimental Search for Quantum Gravity 2010, recording at videos.nordita.org/programs/esqg2010/2010-07-14-bojowald.wmv

[28] R. Schutzhold and W. G. Unruh, JETP Lett. 78, 431 (2003) [Pisma Zh. Eksp. Teor. Fiz. 78, 899 (2003)] [arXiv:gr-qc/0308049]; S. Hossenfelder, arXiv:0912.0090 [gr-qc]; S. Hossenfelder, Phys. Rev. Lett. 104, 140402 (2010) [arXiv:1004.0418 [hep-ph]]. 
[29] U. Jacob, F. Mercati, G. Amelino-Camelia and T. Piran, arXiv:1004.0575 [astro-ph.HE]; L. Smolin, arXiv:1004.0664 [gr-qc]; S. Hossenfelder, arXiv:1005.0535 [gr-qc]; G. AmelinoCamelia, M. Matassa, F. Mercati and G. Rosati, arXiv:1006.2126 [gr-qc]; S. Hossenfelder, arXiv:1006.4587 [gr-qc]; L. Smolin, arXiv:1007.0718 [gr-qc]; S. Hossenfelder, arXiv: 1008.1312 [gr-qc].

[30] X. Calmet, S. Hossenfelder and R. Percacci, arXiv:1008.3345 [gr-qc].

[31] G. Amelino-Camelia, Int. J. Mod. Phys. D 12, 1211 (2003) [arXiv:astro-ph/0209232].

[32] G. Amelino-Camelia, Symmetry 2, 230 (2010) [arXiv:1003.3942 [gr-qc]].

[33] R. D. Sorkin, arXiv:gr-qc/0309009

[34] L. Bombelli and D. A. Meyer, Phys. Lett. A 141, 226 (1989).

[35] L. Bombelli, J. Henson and R. D. Sorkin, Mod. Phys. Lett. A 24, 2579 (2009) [arXiv:gr-qc/0605006].

[36] F. Dowker, J. Henson and R. D. Sorkin, Mod. Phys. Lett. A 19, 1829 (2004) [arXiv:gr-qc/0311055]; L. Philpott, F. Dowker and R. D. Sorkin, Phys. Rev. D 79, 124047 (2009) [arXiv:0810.5591 [gr-qc]].

[37] W. Heisenberg, Annalen der Physik, 32, 20-33, (1938).

[38] L. J. Garay, Int. J. Mod. Phys. A 10, 145 (1995) [arXiv:gr-qc/9403008]; Y. J. Ng, Mod. Phys. Lett. A 18, 1073 (2003) [arXiv:gr-qc/0305019].

[39] D. J. Gross and P. F. Mende, Nucl. Phys. B 303, 407 (1988); K. Konishi, G. Paffuti and P. Provero, Phys. Lett. B 234, 276 (1990). D. Amati, M. Ciafaloni and G. Veneziano, Phys. Lett. B 216, 41 (1989); T. Yoneya, Mod. Phys. Lett. A 4, 1587 (1989).

[40] C. Rovelli, Living Rev. Rel. 1, 1 (1998) [arXiv:gr-qc/9710008]; A. Perez, Class. Quant. Grav. 20, R43 (2003) [arXiv:gr-qc/0301113]; T. Thiemann, Lect. Notes Phys. 631, 41 (2003) [arXiv:gr-qc/0210094]; A. Ashtekar and J. Lewandowski, Class. Quant. Grav. 21, R53 (2004).

[41] M. R. Douglas and N. A. Nekrasov, Rev. Mod. Phys. 73, 977 (2001).

[42] E. P. Wigner, Rev. Mod. Phys. 29, 255 (1957); H. Salecker and E. P. Wigner, Phys. Rev. 109, 571 (1958); J. D. Barrow, Phys. Rev. D 54, 6563 (1996); N. Sasakura, Prog. Theor. Phys. 102, 169 (1999) [arXiv:hep-th/9903146]; F. Scardigli, Phys. Lett. B 452, 39 (1999) |arXiv:hep-th/9904025]; T. Padmanabhan, T. R. Seshadri and T. P. Singh, Int. J. Mod. Phys. A 1, 491 (1986); X. Calmet, M. Graesser and S. D. H. Hsu, Phys. Rev. Lett. 93, 211101 (2004) [arXiv:hep-th/0405033]; V. Daftardar and N. Dadhich, Int. J. Mod. Phys. A 1, 731 (1986); Y. J. Ng and H. van Dam, Int. J. Mod. Phys. A 20, 1328 (2005) [arXiv:gr-qc/0403057]; Y. J. Ng and H. van Dam, Annals N. Y. Acad. Sci. 755, 579 (1995) [arXiv:hep-th/9406110].

[43] A. Kempf, G. Mangano and R. B. Mann, Phys. Rev. D 52, 1108 (1995) [arXiv:hep-th/9412167]; M. Maggiore, Phys. Lett. B 319, 83 (1993) [arXiv:hep-th/9309034]; M. Maggiore, Phys. Rev. D 49, 5182 (1994) [arXiv:hep-th/9305163]; A. Camacho, Int. J. Mod. Phys. D 12, 1687 (2003) [arXiv:gr-qc/0305052]; I. Dadic, L. Jonke and S. Meljanac, Phys. Rev. D 67, 087701 (2003) |arXiv:hep-th/0210264]; F. Brau, J. Phys. A 32, 7691 (1999) [arXiv:quant-ph/9905033]; R. Akhoury and Y. P. Yao, Phys. Lett. B 572, 37 (2003) [arXiv:hep-ph/0302108]; S. Hossenfelder et al, Phys. Lett. B 575, 85 (2003) [arXiv:hep-th/0305262]; S. Hossenfelder, Phys. Rev. D 70, 105003 (2004) [arXiv:hep-ph/0405127]; M. Kober, [arXiv:1008.0154 [physics.gen-ph]].

[44] S. Hossenfelder, Mod. Phys. Lett. A 19, 2727 (2004) [arXiv:hep-ph/0410122]. 
[45] A. Camacho and A. Macias, Gen. Rel. Grav. 39, 1175 (2007) [arXiv:gr-qc/0702150]; S. Das, S. Ghosh and D. Roychowdhury, Phys. Rev. D 80, 125036 (2009) [arXiv:0908.0413 [hep-th]]; S. Das and D. Roychowdhury, Phys. Rev. D 81, 085039 (2010) [arXiv:1002.0192 [hep-th]].

[46] S. Hossenfelder, Class. Quant. Grav. 23, 1815 (2006) [arXiv:hep-th/0510245].

[47] S. Hossenfelder, Phys. Rev. D 73, 105013 (2006) [arXiv:hep-th/0603032].

[48] J. R. Ellis, J. S. Hagelin, D. V. Nanopoulos and M. Srednicki, Nucl. Phys. B 241, 381 (1984); L. H. Ford, Phys. Rev. D 51, 1692 (1995) arXiv:gr-qc/9410047]; L. J. Garay, Int. J. Mod. Phys. A 14, 4079 (1999) [arXiv:gr-qc/9911002]; N. E. Mavromatos, Lect. Notes Phys. 669, 245 (2005) [arXiv:gr-qc/0407005]; N. E. Mavromatos and S. Sarkar, arXiv:hep-ph/0612193.

[49] Y. Bonder and D. Sudarsky, Rept. Math. Phys. 64, 169 (2009) [arXiv:0811.1229 [gr-qc]].

[50] F. Markopoulou, J. Phys. Conf. Ser. 67, 012019 (2007) [arXiv:gr-qc/0703027].

[51] T. Konopka, F. Markopoulou and L. Smolin, arXiv:hep-th/0611197; T. Konopka, F. Markopoulou and S. Severini, Phys. Rev. D 77, 104029 (2008) [arXiv:0801.0861 [hepth]]; T. Konopka, Phys. Rev. D 78, 044032 (2008) [arXiv:0805.2283 [hep-th]]; F. Conrady, arXiv:1009.3195 [gr-qc].

[52] J. Magueijo, L. Smolin and C. R. Contaldi, Class. Quant. Grav. 24, 3691 (2007) [arXiv:astro-ph/0611695|.

[53] M. Bojowald, Living Rev. Rel. 8, 11 (2005) [arXiv:gr-qc/0601085].

[54] M. Bojowald, Phys. Rev. Lett. 86, 5227 (2001) [arXiv:gr-qc/0102069].

[55] A. Ashtekar, T. Pawlowski and P. Singh, Phys. Rev. Lett. 96, 141301 (2006) [arXiv:gr-qc/0602086].

[56] M. Bojowald, G. M. Hossain, M. Kagan and S. Shankaranarayanan, Phys. Rev. D 79, 043505 (2009) [arXiv:0811.1572 [gr-qc]].

[57] E. J. Copeland, D. J. Mulryne, N. J. Nunes and M. Shaeri, Phys. Rev. D 79, 023508 (2009) [arXiv:0810.0104 [astro-ph]]; J. Grain and A. Barrau, Phys. Rev. Lett. 102, 081301 (2009) [arXiv:0902.0145 [gr-qc]]; J. Mielczarek, Phys. Rev. D 81, 063503 (2010) arXiv:0908.4329 [gr-qc]].

[58] M. Gasperini, "Elements of string cosmology," Cambridge University Press, Cambridge (2007).

[59] M. Gasperini and G. Veneziano, Astropart. Phys. 1, 317 (1993) [arXiv:hep-th/9211021]; Mod. Phys. Lett. A 8, 3701 (1993) [arXiv:hep-th/9309023]; Phys. Rev. D 50, 2519 (1994) [arXiv:gr-qc/9403031].

[60] M. Gasperini and G. Veneziano, Phys. Rept. 373, 1 (2003) [arXiv:hep-th/0207130].

[61] R. H. Brandenberger and C. Vafa, Nucl. Phys. B 316, 391 (1989); A. A. Tseytlin and C. Vafa, Nucl. Phys. B 372, 443 (1992) [arXiv:hep-th/9109048]; S. Alexander, R. H. Brandenberger and D. A. Easson, Phys. Rev. D 62, 103509 (2000) [arXiv:hep-th/0005212]; A. Nayeri, R. H. Brandenberger and C. Vafa, Phys. Rev. Lett. 97, 021302 (2006) [arXiv:hep-th/0511140]; Int. J. Mod. Phys. A 22, 3621 (2007) [arXiv:hep-th/0608121]; arXiv:0808.0746 [hep-th], publ. in “String Cosmology,” ed. by J. Erdmenger (Springer, Berlin, 2009).

[62] T. Battefeld and S. Watson, Rev. Mod. Phys. 78, 435 (2006) [arXiv:hep-th/0510022. 
[63] J. Khoury, B. A. Ovrut, P. J. Steinhardt and N. Turok, Phys. Rev. D 64, 123522 (2001) [arXiv:hep-th/0103239]; J. Khoury et al, Phys. Rev. D 65, 086007 (2002) [arXiv:hep-th/0108187]; P. J. Steinhardt and N. Turok, Phys. Rev. D 65, 126003 (2002) |arXiv:hep-th/0111098|; E. I. Buchbinder, J. Khoury and B. A. Ovrut, Phys. Rev. D 76, 123503 (2007) [arXiv:hep-th/0702154].

[64] C. P. Burgess et al, JHEP 0107, 047 (2001) [arXiv:hep-th/0105204]; F. Quevedo, Class. Quant. Grav. 19, 5721 (2002) [arXiv:hep-th/0210292]; S. Kachru et al, JCAP 0310, 013 (2003) [arXiv:hep-th/0308055]; S. Kachru et al, Phys. Rev. D 68, 046005 (2003) [arXiv:hep-th/0301240].

[65] S. P. Patil and R. H. Brandenberger, JCAP 0601, 005 (2006) [arXiv:hep-th/0502069].

[66] R. Brandenberger, Y. K. Cheung and S. Watson, JHEP 0605, 025 (2006) [arXiv:hep-th/0501032].

[67] N. Arkani-Hamed, S. Dimopoulos and G. R. Dvali, Phys. Lett. B 429, 263 (1998) |arXiv:hep-ph/9803315|; I. Antoniadis, N. Arkani-Hamed, S. Dimopoulos and G. R. Dvali, Phys. Lett. B 436, 257 (1998) [arXiv:hep-ph/9804398]; N. Arkani-Hamed, S. Dimopoulos and G. R. Dvali, Phys. Rev. D 59, 086004 (1999) [arXiv:hep-ph/9807344].

[68] H. C. Cheng, arXiv:1003.1162 [hep-ph].

[69] M. Masuda and M. Sasaki, Phys. Rev. Lett. 102, 171101 (2009) [arXiv:0904.1834 [hep-ex]].

[70] L. Randall and R. Sundrum, Phys. Rev. Lett. 83, 3370 (1999) [arXiv:hep-ph/9905221]; L. Randall and R. Sundrum, Phys. Rev. Lett. 83, 4690 (1999) [arXiv:hep-th/9906064].

[71] R. Maartens and K. Koyama, Living Rev. Rel. 13, 5 (2010) [arXiv:1004.3962 [hep-th]].

[72] T. W. B. Kibble, J. Phys. A 9, 1387 (1976); Phys. Rept. 67, 183 (1980).

[73] A. Achucarro and C. J. A. Martins, arXiv:0811.1277 [astro-ph].

[74] M. Majumdar and A. Christine-Davis, JHEP 0203, 056 (2002) |arXiv:hep-th/0202148]; S. Sarangi and S. H. H. Tye, Phys. Lett. B 536, 185 (2002) [arXiv:hep-th/0204074]; A. C. Davis and T. W. B. Kibble, Contemp. Phys. 46, 313 (2005) [arXiv:hep-th/0505050].

[75] K. Nakamura [Particle Data Group], J. Phys. G 37, 075021 (2010).

[76] S. Cullen and M. Perelstein, Phys. Rev. Lett. 83, 268 (1999) [arXiv:hep-ph/9903422].

[77] S. Hannestad and G. Raffelt, Phys. Rev. Lett. 87, 051301 (2001) arXiv:hep-ph/0103201].

[78] S. Hannestad and G. G. Raffelt, Phys. Rev. Lett. 88, 071301 (2002) [arXiv:hep-ph/0110067].

[79] R. C. Myers and M. Pospelov, Phys. Rev. Lett. 90, 211601 (2003) arXiv:hep-ph/0301124].

[80] L. Maccione, S. Liberati, A. Celotti and J. G. Kirk, JCAP 0710, 013 (2007) arXiv:0707.2673 [astro-ph]].

[81] G. Amelino-Camelia, J. R. Ellis, N. E. Mavromatos, D. V. Nanopoulos and S. Sarkar, Nature 393, 763 (1998) [arXiv:astro-ph/9712103].

[82] M. Ackermann et al. [Fermi GBM/LAT Collaborations], Nature 462, 331 (2009) [arXiv:0908.1832 [astro-ph.HE]].

[83] M. Biesiada and A. Piorkowska, Mon. Not. Roy. Astron. Soc. 396, 946 (2009) [arXiv:0712.0941 [astro-ph]].

[84] T. Damour and A. Vilenkin, Phys. Rev. D 71, 063510 (2005) arXiv:hep-th/0410222;; 
[85] E. O'Callaghan, S. Chadburn, G. Geshnizjani, R. Gregory and I. Zavala, Phys. Rev. Lett. 105, 081602 (2010) [arXiv:1003.4395 [hep-th]]; E. O'Callaghan, S. Chadburn, G. Geshnizjani, R. Gregory and I. Zavala, JCAP 1009, 013 (2010) [arXiv:1005.3220 [hep-th]].

[86] R. T. Thompson and L. H. Ford, Phys. Rev. D 74, 024012 (2006) [arXiv:gr-qc/0601137].

[87] M. Gasperini and M. Giovannini, Phys. Rev. D 47, 1519 (1993) [arXiv:gr-qc/9211021]; R. Brustein, M. Gasperini, M. Giovannini and G. Veneziano, Phys. Lett. B 361, 45 (1995) [arXiv:hep-th/9507017].

[88] M. Kamionkowski, A. Kosowsky and A. Stebbins, Phys. Rev. Lett. 78, 2058 (1997) |arXiv:astro-ph/9609132].

[89] L. A. Boyle, P. J. Steinhardt and N. Turok, Phys. Rev. D 69, 127302 (2004) [arXiv:hep-th/0307170|.

[90] R. H. Brandenberger, A. Nayeri, S. P. Patil and C. Vafa, Phys. Rev. Lett. 98, 231302 (2007) [arXiv:hep-th/0604126].

[91] J. Mielczarek, T. Cailleteau, J. Grain and A. Barrau, Phys. Rev. D 81, 104049 (2010) arXiv:1003.4660 [gr-qc]].

[92] Y. Z. Ma, W. Zhao and M. L. Brown, arXiv:1007.2396 [astro-ph.CO].

[93] C. Ringeval, Adv. Astron. 2010, 380507 (2010) [arXiv:1005.4842 [astro-ph.CO]].

[94] C. R. Contaldi, F. Dowker and L. Philpott, Class. Quant. Grav. 27, 172001 (2010) [arXiv:1001.4545 [gr-qc]].

[95] D. M. Mattingly, L. Maccione, M. Galaverni, S. Liberati and G. Sigl, JCAP 1002, 007 (2010) [arXiv:0911.0521 [hep-ph]].

[96] J. Christian, Phys. Rev. D 71, 024012 (2005) [arXiv:gr-qc/0409077].

[97] J. Christian, Phys. Rev. Lett. 95, 160403 (2005) [arXiv:quant-ph/0503001].

[98] V. A. Kostelecky and M. Mewes, Phys. Rev. D 70, 031902 (2004) [arXiv:hep-ph/0308300].

[99] V. Barger, D. Marfatia and K. Whisnant, Phys. Lett. B 653, 267 (2007) arXiv:0706.1085 [hep-ph]].

[100] S. Hollenberg, O. Micu and H. Pas, Prog. Part. Nucl. Phys. 64, 193 (2010) arXiv:0911.1018 [hep-ph]].

[101] G. Amelino-Camelia and L. Smolin, Phys. Rev. D 80, 084017 (2009) arXiv:0906.3731 [astro-ph.HE]].

[102] C. J. Hogan, arXiv:0905.4803 [gr-qc].

[103] Y. Bonder and D. Sudarsky, AIP Conf. Proc. 1256, 157 (2010) [arXiv:1003.5245 [gr-qc]].

[104] R. Adler et al. [CPLEAR collaboration], Phys. Lett. B 364, 239 (1995) [arXiv:hep-ex/9511001]; F. Ambrosino et al. [KLOE Collaboration], Phys. Lett. B 642, 315 (2006) [arXiv:hep-ex/0607027].

[105] D. N. Spergel et al. [WMAP Collaboration], Astrophys. J. Suppl. 170, 377 (2007) |arXiv:astro-ph/0603449].

[106] C. Prescod-Weinstein and L. Smolin, Phys. Rev. D 80, 063505 (2009) arXiv:0903.5303 [hepth]].

[107] S. H. S. Alexander, arXiv:0911.5156 [hep-ph]. 
[108] R. D. Sorkin, Int. J. Theor. Phys. 36, 2759 (1997) [arXiv:gr-qc/9706002].

[109] Y. K. Cheung, K. Savvidy and H. C. Kao, arXiv:astro-ph/0702290; Y. K. Cheung and F. Xu, arXiv:0810.2382 [hep-th].

[110] R. H. Brandenberger and C. Vafa, Nucl. Phys. B 316, 391 (1989). 